ISSN 1991-8631

Original Paper

http://indexmedicus.afro.who.int

\title{
Essai de conservation de la tomate par la technique de la déshydratation imprégnation par immersion (DII)
}

\author{
Michelline AGASSOUNON DJIKPO TCHIBOZO ${ }^{1}$, Selma GOMEZ ${ }^{1}$, \\ Fidèle Paul TCHOBO ${ }^{2}$, Mohamed M. SOUMANOU ${ }^{2 *}$ et Fatiou TOUKOUROU ${ }^{1}$ \\ ${ }^{1}$ Laboratoire de Microbiologie et des Technologies Alimentaires (LAMITA), \\ Faculté des Sciences et Techniques (FAST), Université d'Abomey-Calavi (UAC), Bénin. \\ ${ }^{2}$ Unité de Recherche en Génie Enzymatique et Alimentaire, Laboratoire d'Etudes et de Recherches en Chimie \\ Appliquée, Ecole Polytechnique d'Abomey-Calavi (EPAC), Université d'Abomey-Calavi (UAC), \\ 01 BP 2009, Cotonou, Bénin. \\ *Auteur correspondant, E-mail : msoumanoufr@yahoo.fr, mohamed.soumanou@epac.uac.bj
}

\section{RESUME}

La Déshydratation Imprégnation par Immersion (DII) est une technique de conservation à long terme déjà appliquée sur certains fruits comme la mangue, l'ananas, la banane, etc. Le présent travail est une application de la DII à la conservation de la tomate (Lycopercicon esculentum Mill.) variété locale Tounvi largement cultivée au Bénin. Ainsi, dans les conditions expérimentales de production, une augmentation de la teneur en matière sèche soluble de $5 \%$ à $80,79 \%$ sous l'effet de la chaleur au cours de l'obtention des tomates séchées, s'est traduite par une élévation subséquente de la teneur en cendres qui passe de $0,51 \%$ pour le produit frais à $0,75 \%$ à la fin de la DII. L'acidité des tomates également varie de $4,5 \mathrm{~g} / \mathrm{L}$ à $5,72 \mathrm{~g} / \mathrm{L}$, ce qui entraîne une légère diminution du $\mathrm{pH}$ qui passe de 4,36 à 4,12 à la fin de la DII. Nous notons également une diminution de la teneur en vitamine $\mathrm{C}$ qui passe de $335,41 \mathrm{mg} / \mathrm{L}$ (produit frais) à 178,25 mg/L (produit séché), soit une réduction de 53,14\%. Sur le plan microbiologique, pendant une période de sept (7) semaines, une conformité à la norme AFNOR (NFV-08-402) est notée ; ce qui justifie une bonne stabilité des produits, caractérisée par l'absence des Anaérobies Sulfito-Réducteurs (ASR) et la présence de 35 UFC de germe aérobie mésophile/g et de 20 UFC Levures et Moisissures/g de tomates séchées.

(c) 2012 International Formulae Group. All rights reserved.

Mots clés : Tomate, conservation, qualité, stabilité microbiologique.

\section{INTRODUCTION}

La tomate (Lycopercicon esculentum Mill.) est l'une des cultures maraîchères les plus importantes du Bénin. La production annuelle est en constante augmentation, passant de 157. 414 tonnes en 2005 à 159.132 tonnes en 2006, de 163.304 tonnes en 2007 à 156.104 tonnes en 2008 (DPP/MAEP, 2008), ce qui correspond à une croissance de $11 \%$. Sa culture s'impose comme une importante activité génératrice de revenus pour les femmes aussi bien des milieux ruraux que dans les milieux périurbains (DAPS/MDR, 1994), et en particulier celles des Départements du sud du Bénin qui fournissent plus de $80 \%$ de la production nationale. Sur le 
plan nutritionnel, les travaux de Gagnon (1991) avaient révélé que ce fruit est riche en minéraux $(\mathrm{Ca}, \mathrm{Fe}, \mathrm{K}, \mathrm{Mg}, \mathrm{Na}, \ldots)$ et en vitamines $\left(\mathrm{A}, \mathrm{B}_{6}, \mathrm{C}, \mathrm{E}\right)$. Malgré son importance, la production de la tomate demeure inorganisée et on enregistre régulièrement au Bénin, une longue période de pénurie, d'octobre à mai, et une courte période d'abondance de juin à septembre ; ainsi, on note une perte de plus de $40 \%$ (Dossou et al., 2007) de production nationale en saison d'abondance, faute de moyens efficaces de conservation de tomate. La rareté de l'offre en période de soudure contraint à l'importation de tomate fraîche des pays voisins que sont le Nigéria, le Togo et le Burkina-faso; ainsi que de concentré de tomate importé, venant surtout de Chine et Singapour. La transformation industrielle initiée au cours des années 70 avec l'installation d'une usine de concentré de tomate à Natitingou (au nord-ouest du Bénin) ayant échoué, la transformation à petite échelle est aujourd'hui préconisée comme une issue probable (Soulé, 2001). Aussi, la transformation artisanale des tomates, surtout en purée, connaît un accroissement constant depuis le début des années 90, mais elle n'a pu réduire l'importance des pertes au niveau des producteurs pendant la période d'abondance. De plus, les trois techniques de conservation que sont le séchage, le chauffage à l'ébullition et le salage ne sont plus efficaces pour une conservation à long terme de ce fruit-légume ; car ses caractéristiques nutritionnelles et/ou marchandes sont altérées (Vierling, 2003). Il convient donc de rechercher des techniques de conservation permettant de garantir les caractéristiques nutritionnelles et marchandes de la tomate traitée par rapport au fruit frais. Ainsi, le présent travail explore l'opportunité de conserver la tomate fraîche variété Tounvi à travers un prétraitement avant séchage par la technique de Déshydratation par ImmersionImprégnation (DII).

\section{MATERIEL ET METHODES Matériel végétal}

Les études ont porté sur les fruits de tomate (Lycopercicon esculentum Mill.), variété locale "Tounvi ", reconnue par la présence de 3 à 5 lobes, de forme aplatie et de couleur rouge intense, achetés au Bénin dans le marché du St Michel à Cotonou. " Elle appartient à la classe des variétés à petit fruit avec un poids moyen de $41,8 \mathrm{~g} /$ fruit (Fagbohoun et Kiki, 2000).

\section{Echantillonnage, traitement préliminaire des fruits et autres matériels utilisés}

Un panier d'environ $15 \mathrm{~kg}$ de tomates mûres, fermes et saines, conservant leur pédoncule a été sélectionné au hasard, pour servir à la mise au point des différents lots de produits, pour les essais. Dès la réception, les fruits ont été étalés sur des étalages assainis avec de l'eau javellisée $0,08 \%$ et aérés; puis un triage manuel des fruits mûrs de tomate achetés a constitué à séparer les déchets des fruits de couleur rouge uniforme et ferme. Une fois les tomates triées, elles ont été lavées à grande eau avec une éponge dans une bassine; puis désinfectées par trempage pendant $30 \mathrm{~min}$ avec de l'eau javellisée à $0,08 \%$ et rincées une dernière fois à grande eau puis égouttées (Figure 1). Les fruits ont été répartis en 3 lots qui sont codés : lot I (tomate entière avec épicarpe); lot II (tomate sans épicarpe); lot III (tomate séchée). Seules les tomates mûres, fermes et saines ont été sélectionnées pour les essais. La solution osmotique a été préparée à des concentrations variables avec le sel de cuisine. Des boîtes de conserve en verre, à fermeture étanche (recyclées) ont servi à recueillir les fruits et la solution d'imprégnation, ainsi qu'à leur 
conditionnement. Le papier filtre est utilisé pour l'essorage.

\section{Technique de Déshydratation Imprégnation par Immersion (DII)}

Avant la réalisation de cette technique, à partir du sel fin de cuisine, la solution osmotique a été produite avec de l'eau distillée stérile à des concentrations de 100 $\mathrm{g} / \mathrm{L}, 200 \mathrm{~g} / \mathrm{L}$ et $300 \mathrm{~g} / \mathrm{L}$. Il s'agit de la saumure osmotique qui a été laissée sous une agitation pour une bonne homogénéisation, elle a été bouillie à $100{ }^{\circ} \mathrm{C}$ pendant 1 heure, avant d'être utilisée pour le trempage (immersion) des fruits de tomate (Figure 1). Un lot des fruits a été distribué à raison de 720 g par boîte de conserve en verre propre et étuvé à $105{ }^{\circ} \mathrm{C}$, et auxquelles les saumures précédemment préparées à des concentrations de $100 \mathrm{~g} / \mathrm{L}, 200 \mathrm{~g} / \mathrm{L}$ et $300 \mathrm{~g} / \mathrm{L}$ ont été ajoutées, dans un rapport/soluté de 1/10 (M/V). Ensuite, l'ensemble a été hermétiquement fermé, maintenu dans un bain-marie thermo réglable à $60{ }^{\circ} \mathrm{C}$ pendant 12 heures (Figure 1) et sous une agitation manuelle intermittente pour une bonne imprégnation. A la fin de cette étape, les échantillons ont été refroidis, rincés à l'eau distillée stérile, essorés sur du papier filtre, épicarpés et découpés en 2, 4 ou 6 tranches dans le sens de la longueur. Cette opération a permis de vider les tomates du mélange geléepépins et de leur offrir une surface d'échange de chaleur nettement réduite au cours du séchage.

\section{Séchage}

Le dispositif de séchage utilisé est mis au point au Centre Béninois de Recherche Scientifique et Technique (CBRST, Bénin), il est muni d'une pellicule en plastique qui protège les fruits contre les insectes et mouches. Pour la déshydratation les tranches du lot III de tomate ont été soumises au séchage solaire à la température de $45^{\circ} \mathrm{C}$. Des échantillons de tomate témoin (lot II), n'ayant subi aucun prétraitement de DII, ont été séchés dans les mêmes conditions. Le suivi du séchage solaire a été effectué tous les jours pendant 72 heures, par la mesure de la variation de masse (M) des fruits; puis on a déduit la vitesse de séchage $(\mathrm{dM} / \mathrm{dt})$. Après le séchage, le conditionnement a été fait dans des bocaux de $370 \mathrm{~g}$ préalablement lavés et stérilisés. Une fois les bocaux remplis de tomates séchées, ils ont été fermés hermétiquement; puis emballés dans du papier aluminium et conservés à la température ambiante $\mathrm{du}$ laboratoire maintenue à $26{ }^{\circ} \mathrm{C}$ par la climatisation. La Figure 1 illustre les différentes étapes de la DII.

\section{Caractéristiques morphologiques des fruits de tomate}

Avant les traitements préliminaires, les caractéristiques morphologiques ont été déterminées sur une trentaine de fruits de bonne qualité. Cette étude a été exprimée par le nombre de lobes déterminé par comptage pour chaque fruit, le poids moyen du fruit $\left(\mathrm{P}_{\mathrm{m}}\right)$, la masse volumique $\left(\rho_{\mathrm{f}}\right)$ et le coefficient de forme $\left(\mathrm{C}_{\mathrm{f}}\right)$. Le $\mathrm{C}_{\mathrm{f}}$ a été déterminé par la formule suivante déjà appliquée par Fagbohoun et Kiki (2000). Le coefficient de forme permet de distinguer le cultivar auquel appartiennent les fruits utilisés ainsi que de classer les variétés en trois catégories, notamment: $\mathrm{C}_{\mathrm{f}}<0,8$ indique une forme aplatie ; $\mathrm{C}_{\mathrm{f}}>1$ indique une forme allongée et $0,8<\mathrm{C}_{\mathrm{f}}<1$ indique une forme ronde.

$$
\mathrm{C}_{\mathrm{f}}=\frac{\text { Hauteur moyenne du fruit }}{\text { Diamètre moyen du fruit }}
$$

Détermination des teneurs en humidité et des transferts de matières au cours de l'immersion des tomates

Cette étude a concerné la détermination de la teneur en eau; de la perte d'eau à l'instant $\mathrm{t}$; la réduction $\mathrm{du}$ poids après 


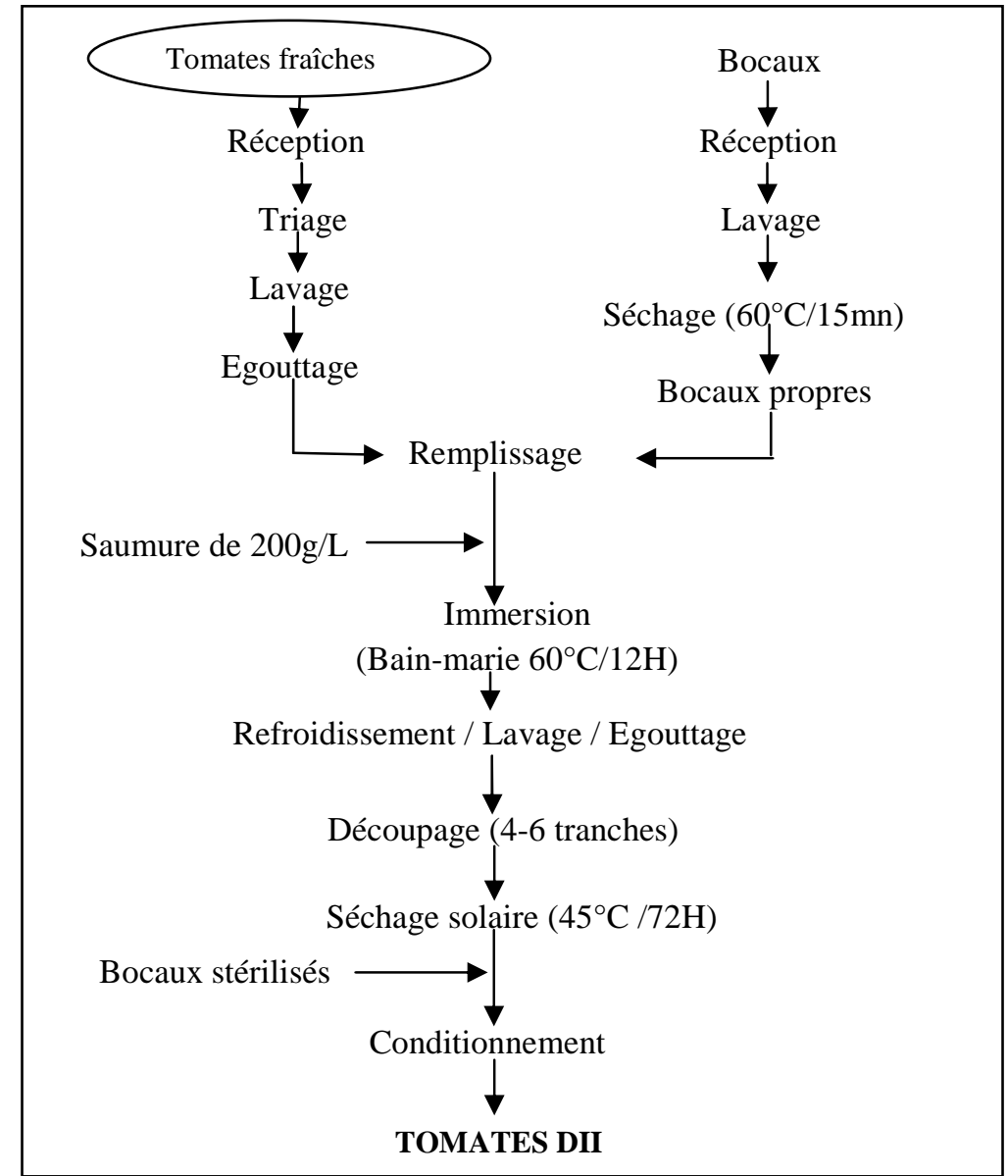

Figure 1 : Diagramme de production des tomates traitées par la DII.

immersion ; le gain de soluté de la solution d'immersion après immersion. La détermination de la teneur en eau a été faite à $105^{\circ} \mathrm{C} / 24$ heures (Agassounon et al., 2007). Pour la détermination des transferts de matières qui ont eu lieu durant le procédé, c'est la technique suivie par Jiokap Nono et al. (2001) qui a été utilisée à travers les formules ci-dessous :

- La perte d'eau à l'instant $\mathrm{t}$ :

$$
\mathrm{PE}_{\mathrm{t}}=\left(\mathrm{H}_{\mathrm{to}}-\mathrm{M}_{\mathrm{t}} / \mathrm{M}_{\mathrm{to}}\right) \times \mathrm{H}_{\mathrm{t}}
$$

- La réduction de poids après immersion :

$$
R P_{t}=\left(M_{t o}-M_{t}\right) / M_{t o}
$$

- Le gain de soluté après immersion :

$$
\mathrm{GS}_{\mathrm{t}}=\mathrm{PE}_{\mathrm{t}}-\mathrm{RP}_{\mathrm{t}}
$$

Avec :

$\mathrm{PE}_{\mathrm{t}}=$ perte d'eau à l'instant $\mathrm{t}$ correspondant à l'après immersion des échantillons

$\mathrm{S}_{\mathrm{t}}=$ masse de sel dans les échantillons après immersion

$\mathrm{GS}_{\mathrm{t}}=$ gain de soluté après immersion

$\mathrm{RP}_{\mathrm{t}}=$ réduction de poids des échantillons après immersion

$\mathrm{M}_{\mathrm{to}}=$ masse des échantillons avant immersion ; $\mathrm{M}_{\mathrm{t}}=$ masse des échantillons après immersion

$\mathrm{H}_{\mathrm{to}}=$ teneur en eau des échantillons avant immersion; $\mathrm{H}_{\mathrm{t}}=$ teneur en eau des échantillons après immersion. 
Détermination des paramètres physicochimiques des différents échantillons de tomates

Les analyses physico-chimiques ont été réalisées aussi bien sur les tomates fraîches que sur des échantillons séchés (DII) suivant des méthodes normalisées.

Le $\mathrm{pH}$ a été mesuré directement à l'aide de l'électrode d'un pHmètre SB70P VWR Symphony de précision relative $\pm 0,02$. La matière sèche soluble (MSS) a été déterminée par la méthode AOAC (1984); les cendres totales ont été déterminées par la méthode AOAC (1984); l'acidité titrable a été dosée par la méthode AFNOR, appliquée aux fruits et légumes et dérivés déjà rapportée par Agassounon et al. (2009). Les teneurs en Vitamine $\mathrm{C}$ ou de l'acide ascorbique ont été dosées par la méthode de Pourmaghi-Azar et al. (1997); les teneurs en pro vitamine A ( $\beta$ carotène) ont été dosées par la méthode officielle AOAC (1984); les teneurs en sucres totaux ont été déterminées par la méthode de Dubois et al. (1965).

Analyses microbiologiques
organoleptiques des tranches de tomates
obtenues par DII
Des tranches de tomates DII (lot III)
ont été soumises à des analyses
microbiologiques et sensorielles avant et au
cours des essais de conservation à $26{ }^{\circ} \mathrm{C}$
pendant 7 semaines. L'évaluation a été faite
toutes les semaines dans des conditions
d'asepsie totale.

\section{Préparation des suspensions mères}

Des prélèvements de tranches de fruits DII ont été broyés dans un moulinex ® à 1500 tours/min. Puis, $10 \mathrm{~g}$ du broyat sont dilués dans $90 \mathrm{ml}$ de solution de tryptone-sel pour l'obtention de la solution mère.

\section{Recherche et dénombrement des germes}

Les échantillons de tomates DII ont été soumis à des contrôles microbiologiques pendant 7 semaines à raison d'un contrôle par semaine. L'étude a été effectuée par ensemencement de la suspension mère d'aliquotes de $1 \mathrm{ml}$ et de ses dilutions décimales de $10^{0}$ à $10^{4}$ sur des milieux appropriés provenant des Laboratoires BioMérieux et Diagnostics Pasteur, selon les méthodes normalisées. Les germes tels que : flore mésophile totale à $30{ }^{\circ} \mathrm{C}$ (germes totaux ; NF V08-051) ; levures et moisissures (ISO 7954); Anaérobies Sulfito-réducteurs (ASR XP V08 - 061) ont été recherchés. Après, les milieux ensemencés ont été incubés dans les conditions optimales de culture ; puis le dénombrement a été réalisé et l'expression des résultats est présentée en Unité Formant Colonie par gramme de produit analysé (UFC/g). Le dénombrement des colonies blanches ou colorées, lisses et crémeuses de levures et des moisissures sous forme poudreuse a été noté après 3 à 5 jours d'incubation. Aussi, quelques colonies de moisissures après isolement ont été identifiées par rapport à leurs caractères culturaux, morphologiques par observation en microscopie optique à l'aide du colorant Lactophénol au bleu coton (Moreau, 1974).

\section{Appréciation des caractéristiques sensorielles}

Les études sensorielles des tomates traitées DII avant et au cours des essais de conservation, ont porté sur l'état physique des échantillons en utilisant les paramètres de la norme NF ISO 5492, 1992, que sont la couleur, l'acidité, le goût (salé ou autres), la texture (consistance), l'odeur, les développements de champignons microscopiques et de mouillabilité. Ainsi, à titre expérimental l'appréciation des caractéristiques sensorielles a été effectuée à chaque étape par un panel de 30 dégustateurs amateurs.

\section{Analyse statistique}

L'évaluation statistique des données a été effectuée par l'analyse de variance avec le test de Student à l'aide du logiciel Minitab. 


\section{RESULTATS}

Caractéristiques morphologiques de la variété de tomates étudiées

Le Tableau 1 présente les caractéristiques morphologiques de tomates fruits (Tounvi) fraîches utilisées. Cette variété de tomate est caractérisée par un coefficient de forme supérieur à 0,8 .

Pertes et gain en soluté au cours de l'immersion des différents échantillons de tomates

La détermination des pertes et gain en soluté, sur les tomates fruits, réalisée avec les trois concentrations de saumure $(100 \mathrm{~g} / \mathrm{L}, 200$ $\mathrm{g} / \mathrm{L}$ et $300 \mathrm{~g} / \mathrm{L}$ ) a donné des résultats présentés dans le Tableau 2. Les résultats obtenus indiquent que la perte d'eau et la réduction du poids des échantillons de tomate analysés sont plus élevées pour la concentration de soluté égale à $200 \mathrm{~g} / \mathrm{L}$ contrairement dans le cas des 2 autres concentrations $(100 \mathrm{~g} / \mathrm{L}$ et $300 \mathrm{~g} / \mathrm{L})$. Mentionnons qu'une faible teneur en eau dans les aliments est un atout pour leur conservation ; ce qui justifie le choix de la concentration égale à $200 \mathrm{~g} / \mathrm{L}$ pour la suite de la présente étude.

\section{Caractéristiques physico-chimiques des} tomates au cours de la conservation par DII

Les teneurs en matière sèche soluble (MSS) varient suivant les échantillons (Tableau 3). La teneur en eau est très élevée, soit une moyenne d'environ $95 \%$ aussi bien pour les fruits entiers que pour les fruits sans épicarpe. La Figure 2 présente les résultats de l'évolution de la masse des échantillons au cours du séchage. La variation de la teneur en cendres est analogue aux résultats de la teneur en matière sèche soluble, soit $0,5 \%$ sur les échantillons frais avec épicarpe et sans épicarpe et $0,75 \%$ sur les échantillons séchés (lot III). Par ailleurs, pour la DII une diminution de la valeur du $\mathrm{pH}$ est enregistrée.
Aussi, à la fin de la DII, les échantillons de tomate ont perdu environ 55,35\% de leur teneur en vitamine $\mathrm{C}$, soit une variation de $32,22 \%$ au début de la DII et $17,83 \%$ à la fin. Quant aux teneurs en $\beta$-carotène, une variation très prononcée allant de 105,91 $\mathrm{mg} / 100 \mathrm{~g}$ pour les tomates fraîches à 68,74 $\mathrm{mg} / 100 \mathrm{~g}$ pour les tomates sans épicarpes, soit $50 \%$ de perte, puis à $12,55 \mathrm{mg} / 100 \mathrm{~g}$ à la fin de la DII, ce qui correspond à $85 \%$ de perte. Par ailleurs, les valeurs obtenues par rapport à l'acidité titrable, le $\mathrm{pH}$ et les teneurs en sucres pour chaque catégorie de tomate sont presque similaires (Tableau 3).

Les taux d'acide citrique sont constants à $0,45 \%$ aussi bien pour les tomates fraîches avec épicarpes que celles sans épicarpes. A la fin de la DII, les échantillons obtenus ont une teneur en acide citrique égale à $0,57 \%$.

\section{Caractéristiques microbiologiques des tomates DII}

Les résultats obtenus par rapport à l'analyse microbiologique réalisée sur les tomates issues de la technique de la DII révèlent une grande stabilité microbiologique des échantillons (Tableau 4). Les valeurs en germes d'altération (champignons microscopiques et flores totales) obtenues varient de 10 à $35 \mathrm{UFC} / g$. Les anaérobies Sulfito-réducteurs sont absents.

\section{Caractéristiques sensorielles des tomates DII}

Le Tableau 5 résume les résultats des paramètres sensoriels tels que perçus par les examinateurs. Il ressort de l'analyse (Tableau 5) que $90 \%$ des personnes soumises aux tests trouvent les tranches de tomates déshydratées très rouges, contre $10 \%$ qui les trouvent moins rouge. Quant au goût, 30\% des dégustateurs trouvent les tomates DII très salées, contre $70 \%$ qui les trouvent simplement salées. Ainsi, $60 \%$ des dégustateurs trouvent les 
tomates DII acides, $16,67 \%$ très acides, $13,33 \%$ non acide et enfin $10 \%$ peu acide. En ce qui concerne l'odeur, $43,33 \%$ des évaluateurs trouvent que les tranches de tomate DII ont une odeur très prononcée, qualifiée d'odeur forte de tomate; contre $36,67 \%$ qui la trouvent prononcée et $16,67 \%$ non prononcée.

Tableau 1 : Caractéristiques physico-morphologiques de la variété de tomate étudiée.

\begin{tabular}{lccc}
\hline Nombre de lobes & $\begin{array}{c}\text { Poids moyens des } \\
\text { fruits } \mathbf{P}_{\mathbf{m}}(\mathbf{e n ~} \mathbf{g})\end{array}$ & $\begin{array}{c}\text { Masse volumique } \\
\text { réelle }\left(\mathbf{g} / \mathbf{c m}^{3}\right)\end{array}$ & $\begin{array}{c}\text { Coefficient de } \\
\text { forme }\left(\mathbf{C}_{\mathbf{f}}\right)\end{array}$ \\
\hline $3 \pm 1$ lobes & $28.06 \pm 1.67$ & $0.95 \pm 0.08$ & 0,82 \\
\hline
\end{tabular}

Tableau 2 : Transferts de matières et de solutés au cours de la phase d'immersion des tomates.

\begin{tabular}{lccc}
\hline Paramètres étudiés & \multicolumn{3}{c}{ Concentration $(\mathbf{g} / \mathbf{L})$} \\
\cline { 2 - 4 }$(\boldsymbol{\%})$ & $\mathbf{1 0 0}$ & $\mathbf{2 0 0}$ & $\mathbf{3 0 0}$ \\
\hline Teneur en Humidité & $94.5 \mathrm{a}$ & $86.19 \mathrm{~b}$ & $93.85 \mathrm{a}$ \\
Perte d'Eau & $0.72 \mathrm{a}$ & $16.54 \mathrm{~b}$ & $2.01 \mathrm{c}$ \\
Réduction du Poids & $0.4 \mathrm{a}$ & $9.13 \mathrm{~b}$ & $1.07 \mathrm{c}$ \\
Gain en Soluté & $0.32 \mathrm{a}$ & $7.4 \mathrm{~b}$ & $0.94 \mathrm{c}$
\end{tabular}

Les valeurs moyennes sur la même ligne avec la même lettre ne sont pas significativement différentes $(\mathrm{p}<0,05)$.

Tableau 3 : Caractéristiques physico-chimiques des tomates.

\begin{tabular}{|c|c|c|c|c|c|c|c|}
\hline $\begin{array}{l}\text { Echantillons } \\
\text { analysés }\end{array}$ & MSS (\%) & $\begin{array}{c}\text { Cendre } \\
(\%)\end{array}$ & $\begin{array}{c}\text { Acidité } \\
\text { (g/L) }\end{array}$ & pH & $\begin{array}{c}\text { Vitamine C } \\
\text { (mg.100g }^{-1} \\
\text { MF) }\end{array}$ & $\begin{array}{c}\text { B-carotène } \\
\left(\text { mg.100g }^{-1}\right. \\
\text { MF) }\end{array}$ & $\begin{array}{c}\text { Sucres } \\
(\%)\end{array}$ \\
\hline $\begin{array}{l}\text { Tomate avec } \\
\text { épicarpe }\end{array}$ & $5,14 a$ & $0,56 a$ & $4,50 \mathrm{a}$ & $4,36 a$ & $33,54 a$ & $105,91 \mathrm{a}$ & $3,49 a$ \\
\hline $\begin{array}{l}\text { Tomate sans } \\
\text { épicarpe }\end{array}$ & $5,08 \mathrm{a}$ & $0,51 \mathrm{a}$ & $4,40 \mathrm{a}$ & $4,35 \mathrm{a}$ & $32,21 \mathrm{a}$ & $68,74 b$ & $3,20 \mathrm{a}$ \\
\hline $\begin{array}{l}\text { Tomates } \\
\text { séchées }\end{array}$ & $80,79 b$ & $0,75 b$ & $5,72 b$ & $4,12 \mathrm{a}$ & $17,83 b$ & $12,55 \mathrm{c}$ & $3,03 \mathrm{a}$ \\
\hline
\end{tabular}


Tableau 4 : Dénombrement et évolution des flores mésophile et fongique avant et au cours de la conservation des tomates DII.

\begin{tabular}{cccc}
\hline $\begin{array}{c}\text { Durée de l'analyse } \\
\text { (semaines) }\end{array}$ & \multicolumn{3}{c}{ Nombre de germes (UFC)/g d'échantillon analysé } \\
\cline { 2 - 4 } & $\begin{array}{c}\text { Germes Aérobies } \\
\text { Mésophiles }\end{array}$ & $\begin{array}{c}\text { Levures et Moisissures } \\
\text { Anaérobies Sulfito- } \\
\text { réducteurs }\end{array}$ \\
\hline$*$ & 25 & 10 & $<1$ \\
$\mathbf{1}$ & 30 & 10 & $<1$ \\
$\mathbf{2}$ & 30 & 10 & $<1$ \\
$\mathbf{3}$ & 35 & 15 & $<1$ \\
$\mathbf{4}$ & 30 & 10 & $<1$ \\
$\mathbf{5}$ & 30 & 15 & $<1$ \\
$\mathbf{6}$ & 35 & 15 & $<1$ \\
$\mathbf{7}$ & 35 & &
\end{tabular}

Tableau 5 : Appréciation sensorielle des consommateurs sur les tomates traitées par la DII.

\begin{tabular}{|c|c|c|c|c|c|c|c|c|c|}
\hline \multicolumn{2}{|c|}{ Couleur } & \multicolumn{2}{|c|}{ Acidité } & \multicolumn{2}{|c|}{ Gout } & \multicolumn{2}{|c|}{ Consistance } & \multicolumn{2}{|c|}{$\overline{\text { Odeur }}$} \\
\hline Niveau & $\%$ & Niveau & $\%$ & Niveau & $\%$ & Niveau & $\%$ & Niveau & $\%$ \\
\hline Très rouge & 90 & Très acide & 16.67 & Très salé & 30 & Très ferme & 100 & $\begin{array}{c}\text { Très } \\
\text { prononcée }\end{array}$ & 43.33 \\
\hline Rouge & 10 & Acide & 60 & Salé & 70 & Ferme & 0 & Prononcée & 36.67 \\
\hline Peu rouge & 0 & Peu acide & 10 & Peu salé & 0 & Peu ferme & 0 & $\begin{array}{c}\text { Peu } \\
\text { prononcée }\end{array}$ & 16.67 \\
\hline Pas rouge & 0 & Pas acide & 13.33 & Pas salé & 0 & Pas ferme & 0 & $\begin{array}{c}\text { Pas } \\
\text { prononcée }\end{array}$ & 0 \\
\hline Indifférent & 0 & Indifférent & 0 & Fade & 0 & Indifférent & 0 & Indifférent & 3.33 \\
\hline
\end{tabular}

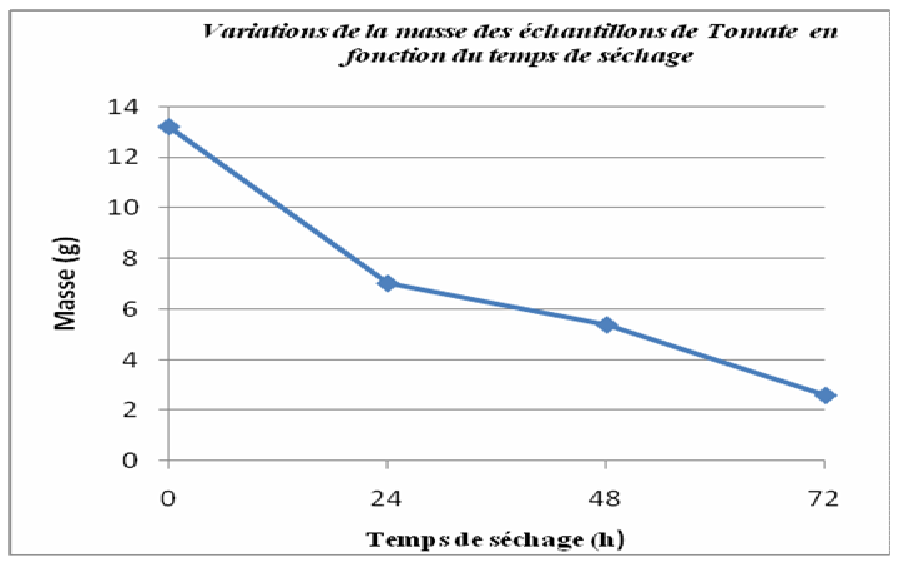

Figure 2 : Evolution de la masse des tranches de tomate au cours du séchage. 


\section{DISCUSSION}

Des résultats relatifs aux caractéristiques morphologiques (Tableau 1) et en se basant sur les observations déjà faites par Fagbohoun et Kiki (2000), nous pouvons dire que les tomates fruits utilisées au cours de nos travaux, appartiennent à la morphotype Tounvi cultivée au Bénin caractérisée par des fruits fortement lobés (4 lobes), gros et légèrement ronds $\left(C_{\mathrm{f}}>0,8\right)$, avec un poids moyen de 28,06 g et une masse volumique de $0,95 \mathrm{~g} / \mathrm{cm}^{3}$. Par rapport à la détermination des pertes et gain en soluté des tomates fruits, pour les différentes concentrations utilisées $(100 \mathrm{~g} / \mathrm{L}, 200 \mathrm{~g} / \mathrm{L}$ et $300 \mathrm{~g} / \mathrm{L})$, la perte d'eau, la réduction du poids et le gain en soluté maximal sont obtenus avec la concentration de $200 \mathrm{~g} / \mathrm{L}$. Ainsi, aux fortes températures avoisinant $60{ }^{\circ} \mathrm{C}$, il pourrait y avoir une dégradation des tissus des fruits, favorisant une incorporation importante de soluté. Selon le principe de l'osmose, la perte d'eau et la réduction de poids à la concentration de 300 $\mathrm{g} / \mathrm{L}$ devraient être supérieures à celles de la solution à $200 \mathrm{~g} / \mathrm{L}$. Cela s'expliquerait par le fait qu'au cours du séjour dans la saumure, les tomates, par le phénomène d'osmose aient perdu une grande quantité d'eau dès les premières heures de l'immersion, ceci grâce au phénomène de diffusion, la concentration intracellulaire en $\mathrm{NaCl}$ deviendra plus élevée que celle de la saumure. L'osmose se déroulera par conséquent dans le sens saumure-tomate jusqu'à la fin du temps d'immersion. A la fin de l'immersion, les échantillons ont une teneur en humidité de 93,85\% sensiblement égale à celle des tomates avant l'immersion (94,86\%). Il est donc possible que la fin de l'immersion à $300 \mathrm{~g} / \mathrm{L}$ coïncide avec le temps d'équilibre du transfert d'eau et de nutriments par osmose. A $100 \mathrm{~g} / \mathrm{L}$, les transferts d'eau et de nutriments sont très faibles, ce qui se traduirait par le faible gain en soluté des échantillons d'une part, la perte d'eau et la réduction de poids qui sont presque nulles. Les teneurs en matière sèche soluble (MSS) inférieures ou égales à 5\% pour les tomates fraîches entières et celles sans épicarpe sont en dessous de la valeur théorique supérieure à 5\% retenues par Fagbohoun et Kiki (1999). Pour la teneur en eau, ce paramètre traduit la grande périssabilité de la tomate et limite son aptitude à l'entreposage à la température ambiante, pendant de très longue période. Ces transferts dépendent essentiellement de certaines variables telles que la forme et la taille des produits immergés, la température (d'imprégnation et de séchage), la concentration et la nature de la solution d'immersion (Jiokap et al., 2000). Par contre, à la fin de la DII, les échantillons de tomates séchés produits ont une teneur en eau et en matières sèches solubles respectivement égale à $80,79 \%$ et $19,21 \%$ (Tableau 3). Il ressort de ces résultats que le traitement par la DII a permis une réduction d'environ $80 \%$ de la teneur en eau des tomates fruits de la variété Tounvi, avec pour conséquence une diminution des risques de périssabilité rapide de tomate. Par ailleurs, la perte en eau très élevée explique également la réduction rapide de la masse des échantillons d'environ $16 \%$ au cours des 72 heures de séchage (Figure 2). La variation de la teneur en vitamine $C$ serait due d'une part aux différentes opérations unitaires de parage des fruits qui ont été réalisées dès la réception des fruits, et d'autre part à l'effet de la température de traitement des tomates au bain marie et surtout du temps de séchage au soleil au cours de la déshydratation. Agassounon et al. (2007), avaient réalisé une étude similaire, mais sur la mangue et avaient rapporté que la vitamine $\mathrm{C}$ est une molécule oxydable très vulnérable au blanchiment. Les études réalisées par 
Dermesonlouoglou et al. (2008) sur la pomme et la tomate avaient montré que le blanchiment limite également certaines pertes nutritionnelles comme la destruction des vitamines, et permet l'élimination de l'air et des gaz inclus dans les tissus végétaux facilitant la réhydratation. Les facteurs de diminution de la teneur de cette vitamine sont la durée de séchage et la présence d'oxygène dans l'air de séchage (Vierling, 2003). Des pertes marquées de vitamine $\mathrm{C}$ ont été aussi enregistrées par Zanoni et al. (1998) lors des études oxydatives sur la tomate. Par contre, lors de la déshydratation osmotique, la vitamine $\mathrm{C}$ étant très hydrosoluble, se répartit entre les morceaux de tomates immergés et le liquide de cuisson ou d'appertisation, ce qui signifierait que le taux différentiel de vitamine C enregistré à la fin de la DII pourrait se retrouver dans la saumure d'immersion à la fin de l'étape de bain-marie (Bilame, 2001). Ainsi, lors de ce type de séchage par imprégnation des fruits et légumes, on note des pertes en vitamine $\mathrm{C}$ liées à sa diffusion vers le liquide externe, et qui augmentent avec la température et la teneur en soluté de la solution d'immersion (Jiokap et al., 2001). Nous pouvons alors déduire qu'en séchage osmotique ou séchage par imprégnation, il est possible d'apporter de la vitamine $\mathrm{C}$ dans la solution osmotique, avec un impact sur la couleur (Plocharski et Konopacka, 2002) et une bonne rétention dans le produit final (environ 80\%) (Konopacka et Markowsky, 2004). En ce qui concerne la $\beta$-carotène, la perte drastique serait due à l'oxydation qui semble être une réaction particulièrement impliquée dans l'évolution de produits séchés (Toor et Savage, 2006). Les travaux de RubioDiaz et al. (2010) ont révélé 1'isomérisation des caroténoïdes au moyen de la spectroscopie infrarouge. Toutefois, Lavelli et al. (1999), trouvent des concentrations en caroténoïdes identiques par rapport à la matière sèche avant et après séchage des tomates, mais Zanoni et al. (1998) ont noté une perte de $10 \%$ seulement après le séchage à $110{ }^{\circ} \mathrm{C}$. D'autres facteurs contribuent essentiellement à la diminution de la teneur en $\beta$-carotènes initiales dans les tomates. Il s'agit d'une part des pertes extrêmement variables ayant lieu lors du transfert d'eau et de matières au cours de l'étape d'immersion dans la saumure et du rinçage des tomates à la sortie de l'immersion. Aussi, le chauffage prolongé semble favoriser l'isomérisation basée sur la déstructuration de la matrice alimentaire permettant une meilleure diffusion. Quant à l'oxydation, elle est bien évidemment favorisée par le contact avec l'air cumulée à une forte activité de l'eau. Elle conduit à une rupture des chaînes hydrocarbonées et à des pertes des qualités organoleptiques et la couleur notamment. Ces valeurs sont en adéquation avec les résultats rapportés par Moresi et Liverotti (1982), qui ont présenté des valeurs de 0,3 à $0,5 \%$ pour les tomates entières. Les valeurs du $\mathrm{pH}$ d'environ 4,3 pour les deux types, sont identiques à celles indiquées par Amoussou (1988) qui a déterminé des valeurs de $\mathrm{pH}$ de 4,0 à 4,4 sur douze variétés de tomate; ces observations concordent également avec celles rapportées par Lamb (1977) sur des produits à base de tomate qui varient de 4,2 à 4,6. A la fin de la DII, nous notons des valeurs de $\mathrm{pH}$ de 4,12 $(\mathrm{pH}<4,2)$. Ce $\mathrm{pH}$ relativement faible des tranches de tomates séchées, est un avantage du point de vue de la stabilité du produit. En effet, ce niveau de $\mathrm{pH}$ pourrait réduire considérablement la nature des microorganismes pouvant se développer sur le produit. Seuls les microorganismes acidophiles, notamment : Levures, Moisissures, Acetobacters et Lactobacillus peuvent s'y développer ; mais pas les Coliformes de type Escherichia coli, puisque 
le $\mathrm{pH}$ minimum requis pour leur développement est de 4,3. La même évolution des résultats a été enregistrée au niveau de la teneur en sucres totaux, où les teneurs initiales sont de 3,50\% et de 3,19\% respectivement pour les tomates fraîches avec épicarpes et celles sans épicarpes. A la fin de la DII, la teneur en sucres totaux est de 3,03\%. Cette situation serait due au fait que le sucre fermentescible, présent à l'intérieur des cellules de tomate, aurait subit une fermentation assez poussée, due à la chaleur de séchage des tranches de tomates, mettant en jeu une masse non négligeable de molécules de sucres représentées par la différence de $5 \mathrm{mg}$ notée à la fin de la DII. Par ailleurs, ce potentiel phénomène de fermentation expliquerait, dans les mêmes conditions l'augmentation de l'acidité relevée à la fin de la DII ainsi que la diminution du pH enregistrée. Pour la stabilité microbiologique des échantillons (lot III) observée durant les 7 semaines, cette observation serait due à la qualité hygiénique des matières premières (tomates fruits), aux conditions hygiéniques de manipulation des fruits au cours des essais de la DII et surtout aux bonnes pratiques de production au cours $\mathrm{du}$ séchage et du conditionnement. Les tomates séchées, conditionnées dans des flacons de verre préalablement stérilisés, emballées dans du papier aluminium, puis soumises à une conservation à température ambiante au laboratoire, paraissent stables, même au-delà d'un mois de conservation. La recherche des Anaérobies Sulfito-réducteurs (ARS) n'a révélé la présence d'aucun de ces germes alors que la norme en tolère $10 / g$ de produit sec. Ceci témoigne d'un bon niveau de maîtrise des paramètres du traitement thermique, puisque les ASR sont les germes responsables de la putréfaction des conserves de produits faiblement acides et ayant subi un sous traitement thermique. Pour ce qui est des études sensorielles sur les tomates DII, les points de vue des examinateurs sont très variables suivant les paramètres retenus (couleur, acidité, goût, consistance et odeur) comme cela s'est fait remarqué à travers les études de production de concentré de tomate réalisées au Bénin par Dossou et al. (2007).

\section{Conclusion}

L'application de la DII sur la tomate constitue des atouts pour le produit final surtout par les gains en matières sèches et en acidité. Toutefois, les pertes enregistrées en vitamines $\mathrm{C}$ et $\beta$-carotènes par rapport au produit frais mettent en évidence que des études doivent être poursuivies pour une meilleure application de cette technique sur la tomate. Aussi, des études devront donc se poursuivre pour l'amélioration de la qualité nutritionnelle du produit fini. L'application d'un double bain marie à différentes concentrations de $\mathrm{NaCl}$ à $30 \%$ et $60 \%$ ou le démarrage du traitement à partir de $85{ }^{\circ} \mathrm{C}$ en laissant décroître la température pendant 24 heures lors de la phase d'imprégnation sont des pistes à explorer.

\section{REFERENCES}

Agassounon DTM, Toukourou F, Gandonou C, Adeoti AZK, Youssouf MC. 2007. Essais de conservation de la mangue à taux d'humidité élevé par la technologie des barrières. Rev. Sci. Med., 5: 53-58.

Agassounon DTM, Ahissou H, Ahanhanzo C, Toukourou F. 2009. Appréciation des qualités microbiologiques et nutritionnelles de la boisson "bissap" issue de la technologie traditionnelle améliorée. J. Rech. Sci. Univ. Lomé, 11(1): 11-19.

Amoussou LF. 1988. Etude des possibilités de production de variétés de tomate 
(Lycopercon esculentum Mill.) de contre saison dans la zone périurbaine de Cotonou. Thèse d'ingénieur agronome, FSA/UNB, Cotonou, Bénin, 150 p.

AOAC. 1984. Official Methods of Analysis Chemists. Airlington: Virginia, USA.

Bilame AF. 2001. Application du procédé de déshydratation impregnation par immersion à la conservation de la tomate (Lycospersion esculentum L.). Mémoire de Maîtrise en Biologie Appliquée, Faculté des Sciences, Université de Ngaoundéré, Cameroun, 44p.

DAPS/MDR. 1994. Filière tomate. Rapport d'étude, Ministère du Développement Rural, République du Bénin, Cotonou, 13 p.

Dermesonlouoglou EK, Pourgouri S, Taoukis PS. 2008. Kinetic study of the effect of the osmotic dehydration pre-treatment to the shelf life of frozen cucumber. Innovative Food Sci. Emerg. Technol., 9: 542-549.

Dossou J, Soulé I, Montcho M. 2007. Evaluation des caractéristiques physicochimiques et sensorielles de la purée de tomate locale produite à petite échelle au Bénin. Tropicultura, 25(2): 119-125.

DPP/MAEP. 2008. Annuaire statistique des productions agricoles. Ministère de 1'Agriculture, de 1'Elevage et de la Pêche, République du Bénin.

Dubois M, Gilles K, Hamilton JK, Rebers PA, Smith F. 1965. Colorimetric method for determination of sugar and related substances. Anal. Chem., 28: 350-356.

Fagbohoun O, Kiki D. 1999. Aperçu sur les principales variétés de tomate locales cultivées dans le sud du Bénin. Bulletin de la Recherche Agronomique du Bénin, 24: 10-21.

Fagbohoun O, Kiki D. 2000. Caractérisation des variétés de tomates locales et développement des technologies appropriées de transformation, PTAA/INRAB, Cotonou. Bénin, 1-18.

Jiokap NY, Nuadje GB, Raoult-Wack AL, Giroux F. 2000. DehydratingImpregnation Soaking (DIS) process of mango pieces (Mangifera indica): solution temperature and concentration effects on the kinetics of certain fruit components. Fruits, 56(3): 169-177.

Jiokap NY, Giroux F, Cuq B, Raoult-Wack AL. 2001. Etudes des paramètres de contrôle et de commande du procédé de déshydratation imprégnation par immersion, sur système probatoire automatisé: application au traitement de pommes "Golden". J. Food Eng., 50(4): 203-210.

Konopacka D, Markowsky J. 2004. Retention of ascorbic acid during apple chips production and storage. Polish J. Food Nutr. Sci., 13(54): 237-241.

Lamb FC. 1977. Tomato products. National Canners Association; Washington CC, Bulletin 27, p. 2.

Lavelli V, Hippeli S, Peri C, Elstner EF. 1999. Evaluation of radical scavenging activity of fresh and airdried tomatoes by three model reactions. J. Agric. Food Chem., 47(9): 3826-3831.

Moreau C. 1974. Moisissures Toxiques dans l'Alimentation ( $2^{\text {ème }}$ édn). Masson: Paris, France; 1-100.

Moresi M, Liverotti C. 1982. Economics study of tomato paste production. Journal of Food Technology, 17(2): 177-192.

Plocharski W, Konopacka D. 2002. Method of manufacturing diet chips of vegetables and fruits. In Brevet WO2002074102-A1.

Pourmaghi-Azar MH, Ojani R. 1997. A selective catalytic voltammetric determination of vitamin $\mathrm{C}$ in pharmaceutral preparations and complex 
matrices of fresh fruit juices. Talanta, 44: 297-303.

Rubio-Diaz DE, Santos A, Francis DM, Rodriguez-Saona LE. 2010. Carotenoid stability during production and storage of tomato juice made from tomatoes with diverse pigment profiles measured by infrared spectroscopy. J. Agric. Food Chem., 58(15): 8692-8698.

Soulé I. 2001. Etude conceptuelle d'une technologie appropriée de fabrication et de conservation de purée de tomate à petite échelle au Bénin. Thèse d'ingénieur agronome, option Nutrition et
Sciences Alimentaires, FSA/UAC, République du Bénin, 125 p.

Toor RK, Savage GP. 2006. Effect of semidrying on the antioxidant components of tomatoes. Food Chem., 94(1): 90-97.

Vierling E. 2003. Aliments et boissons : Filières et produits. In Biosciences et Techniques (2 è édn). Edition Doin; 270p.

Zanoni B, Peri C, Nani R, Lavelli V. 1998. Oxidative heat damage of tomato halves as affected by drying. Food Res. Int., 31(5): 395-401. 